\title{
Editorial: Special issue-emerging technologies and operational analytics
}

\author{
Arnab Adhikari · Adrija Majumdar
}

Published online: 21 January 2021

(C) Indian Institute of Management Calcutta 2021

The rapid technological advancement has opened a plethora of opportunities for firms to improve their operational efficiency and to thrive in this competitive environment by making use of various new technologies.In recent times, the world has experienced the rising importance of analytics in the day to day business operation. Nowadays, firms focus on devising a robust system that results in higher productivity, better capacity utilization, improved flexibility, etc. According to a recent survey conducted by Capgemini Consulting, operational improvement can lead to an increase in the global profit by $\$ 117$ billion. It highlights the importance of operational analytics. Operational analytics can be defined as "the category of business analytics that focuses on measuring the existing and real-time operations of business." The main applications of operational analytics comprise understanding the business phenomenon as well as the market condition, decision making for the improvement of a firm's market position, planning to adapt to the continuous changes in the business word,

A. Adhikari ( $\square)$

Indian Institute of Management Ranchi, Ranchi, Jharkhand, India

e-mail: arnab.a@iimranchi.ac.in

A. Majumdar $(\bowtie)$

Indian Institute of Management Ahmedabad, Ahmedabad,

Gujarat, India

e-mail: adrija@iima.ac.in and facilitating the proper implementation of the proposed operational measures. For this reason, operational analytics involves applications of diverse disciplines such as operations research, statistics, information systems, etc. In general, operations management entails use of scientific analytical methods to formulate optimal decisions for firms and the current growth in technologies have immensely helped this process of decision making (Choi et al. 2018). Still the analytics remain a nascent area specially in the domain of operations management (Mortenson et al. 2015). However, new forms of challenges emerge owing to this transition and we present pertinent research papers in this special issue that addresses some of these issues and methods to overcome.

Furthermore, we are in the age of big data with a high volume, variety, and velocity of data. Processing of such crucial data gives competitive advantage to organisations. Firms are using analytics to discover hidden knowledge from the big data, and improve decision making for both operational aspects and strategic aspects of their company. Current research entails processing of such high volumes and variety of data using state-of-the-art methods and algorithms. Application of emerging technologies in management problems and specially to solve operational issues is the need of the hour and companies are progressing in that direction. Use of sensors to collect data about different equipment and machines, collection of data 
from the social media sites for product development and thereafter use of algorithms to process and generate meaningful insights are some of the important areas of research. Use of analytics to co-ordinate supply chain processes is another active area of research that has strong practical and theoretical importance. In fact, for the current COVID-19 pandemic that has brought in globally unprecedented times, we witness huge application of analytics to solve problems of drug discovery, contract tracing etc.

This special issue comprises seven high quality scholarly works related to operational analytics, which contribute to either theory development or real-life implementation. The studies published in this issue are the result of initial screening and a blinded review process.

The empirical study conducted by Gupta and Basole examines the growth potential of the IT industry encompassing both software products and services and outsourced as well as offshored business processes. The article depicts a holistic picture by assessing the present position of India's IT industry and its prospects. Additionally, it explains societal implications related to the growth potential IT industry such as job creation, business process management, etc.

The paper by Sarangi and Pradhan proposes a conceptual framework to investigate the impact of Information and Communications Technology (ICT) on economic growth. The authors explain how ICT can be useful for economic growth through adequate R\&D support, technical support, deployment of artificial intelligence, autonomic computing applications, transfer of technology to industry and society, enforcement towards acceptance of digitization, etc.

The article by Dhar and Bose presents the effect of Twitter communication on the stock prices of firms in the presence of Covid-19 Pandemic. Here, the authors demonstrate how the organizational communication and the influence of emotions expressed in social media communication in the time of pandemic influence the stock prices of the firms. The detailed analysis yields positive and negative emotions present in the tweets and their relationship with the stock prices. The insights obtained from the study can be useful specially for the practitioners to devise a crisis mitigation strategy.

The article by Mateen et al. focuses on dealing with strategic and operational decision making issues of a firm taking sustainability issues into account. For this purpose, authors propose an analytical methodology describing the vendor managed inventory model considering the reduction of greenhouse emission cost. The article also elaborates different ways of structuring the replenishment policy and achieving the supply chain coordination.

The paper by Biswas et al. explores the impact of the behaviour of the supply chain members on their decision making and the supply chain coordination in the presence of uncertainty. Here, the authors design a risk-averse dyadic manufacturer retailer supply chain using the mean-variance approach. They show how two supply contracts, namely buyback and revenuesharing schemes can be helpful to coordinate the supply chain. Also, the article discusses the effect of risk aversion attitude of the supply chain member on their respective decisions. Further, the authors extend their work from the dyadic to a single-supplier multiple-retailer network and find the existence of pure strategy Nash equilibrium when all the retailers are risk-averse with varying risk attitudes.

The article by Panhalkar and Doye proposes a novel data classification methodology to extract the necessary data to improve the execution speed and space utilization. First, the proposed method incorporates two clustering-based methods for dimensionality reduction and utilizing knowledge from outliers. Next, these methods are integrated into a single supervised and unsupervised decision tree based on cluster analysis pre-processing (DTCAP), which hunts the informative instances from a small, medium, and large dataset. To facilitate the real-life implementation, the methodology has been applied to a real-world dataset of different sizes and exhibits performance improvement by $50 \%$.

The paper by Singh et al. explores an extensive literature review on the application of customer relationship management (CRM) and presents several interesting insights related to the application of supervised and unsupervised learning techniques, the paradigm shift from machine learning to deep learning. Also, the article finds the E-commerce and Telecommunication sectors as the domains with immense growth potential.

We wish to thank all the authors, reviewers, and technical staff for their precious help in the editorial process. Finally, we thank the editors for the opportunity to serve as guest editors for this special issue. 


\section{References}

Choi TM, Wallace SW, Wang Y (2018) Big data analytics in operations management. Product Oper Manag 27(10):1868-1883
Mortenson MJ, Doherty NF, Robinson S (2015) Operational research from taylorism to terabytes: a research agenda for the analytics age. Eur J Oper Res 241(3):583-595

Publisher's Note Springer Nature remains neutral with regard to jurisdictional claims in published maps and institutional affiliations. 